\title{
Rapid species-level identification of vaginal and oral lactobacilli using MALDI-TOF MS analysis and $16 \mathrm{~S}$ rDNA sequencing
}

\author{
Annette Carola Anderson ${ }^{1 *}$, Mohamed Sanunu ${ }^{2}$, Christian Schneider ${ }^{2}$, Andreas Clad ${ }^{3}$, Lamprini Karygianni ${ }^{1}$, \\ Elmar Hellwig ${ }^{1}$ and Ali Al-Ahmad ${ }^{1}$
}

\begin{abstract}
Background: Lactobacillus represents a large genus with different implications for the human host. Specific lactobacilli are considered to maintain vaginal health and to protect from urogenital infection. The presence of Lactobacillus species in carious lesions on the other hand is associated with progressive caries. Despite their clinical significance, species-level identification of lactobacilli still poses difficulties and mostly involves a combination of different phenotypic and genotypic methods. This study evaluated rapid MALDI-TOF MS analysis of vaginal and oral Lactobacillus isolates in comparison to $16 \mathrm{~S}$ rDNA analysis.
\end{abstract}

Results: Both methods were used to analyze 77 vaginal and 21 oral Lactobacillus isolates. The concordance of both methods was at $96 \%$ with five samples discordantly identified. Fifteen different Lactobacillus species were found in the vaginal samples, primarily L. iners, L. crispatus, L. jensenii and L. gasseri. In the oral samples 11 different species were identified, mostly L. salivarius, L. gasseri, L. rhamnosus and L. paracasei. Overall, the species found belonged to six different phylogenetic groups. For several samples, MALDI-TOF MS analysis only yielded scores indicating genus-level identification. However, in most cases the species found agreed with the 165 rDNA analysis result.

Conclusion: MALDI-TOF MS analysis proved to be a reliable and fast tool to identify lactobacilli to the species level. Even though some results were ambiguous while 165 rDNA sequencing yielded confident species identification, accuracy can be improved by extending reference databases. Thus, mass spectra analysis provides a suitable method to facilitate monitoring clinically relevant Lactobacillus species.

\section{Background}

Lactobacilli, gram-positive, highly acidogenic and acid tolerant rods, represent a genus with over 180 known species so far, with lactic acid as their primary fermentation end product $[1,2]$. The human body hosts various Lactobacillus species in different anatomic regions entailing different interactions with the host: e.g. the oral cavity, the intestines and the female genital tract [3-5].

For the latter region lactobacilli play a significant role in the balance of the microbial flora. The healthy female vaginal flora is characterized by different species of lactobacilli and is mostly dominated by one of the following species: Lactobacillus crispatus, Lactobacillus

\footnotetext{
* Correspondence: annette.anderson@uniklinik-freiburg.de

${ }^{1}$ Department of Operative Dentistry and Periodontology,

Albert-Ludwigs-University, Hugstetter Strasse 55, Freiburg, Germany

Full list of author information is available at the end of the article
}

gasseri, Lactobacillus iners and Lactobacillus jensenii [3,6-10]. Within the last decade, culture-independent studies, involving several ethnic groups, revealed differences in vaginal microbiota as well as the absence of lactobacilli in healthy study participants, particularly Hispanic and black women, complicating the understanding of a healthy versus an unhealthy vaginal flora [10-13]. Despite these recent discoveries, it is still acknowledged that these Lactobacillus taxa constitute the predominant genus in the healthy vaginal flora, primarily considering Caucasian women [14-18].

The vaginal lactobacilli play a significant role in protecting their habitat from unwanted invaders. Next to the competition with other species for nutrient supply and adherence to the vaginal epithelium, the production of lactic acid, hydrogen peroxide $\left(\mathrm{H}_{2} \mathrm{O}_{2}\right)$, bacteriocins and other antimicrobial substances inhibit the vaginal colonization by 
more pathogenic microorganisms [19]. Consequently, these species help to prevent the shift of the vaginal microbial composition towards high concentrations of mainly anaerobic bacteria such as Gardnerella vaginalis, Prevotella spp. or Atopobium vaginalis which can lead to a condition known as bacterial vaginosis (BV) [20,21]. Increased bacterial counts for $L$. iners have also been associated with the beginning stages of BV [1,22]. The latter represents the most prevalent vaginal syndrome with a prevalence between $20-50 \%$ for fertile, premenopausal women [23], correlating with an increased risk of various infections. Urinary tract infections, sexually transmitted diseases, late miscarriage and preterm birth are the most frequent among these [24-27]. As a result, the maintenance of a healthy vaginal flora is considered very important. To date, many efforts have been made to develop administrable probiotic Lactobacillus species to reduce the recurrence of infections and promote a healthy vaginal flora $[28,29]$. Some of these probiotic lactobacilli have shown promising results in treating and preventing $\mathrm{BV}$ as well as preventing urinary tract infections [30-33].

In addition, high counts of Lactobacillus species are not only present in the vaginal and intestinal flora, but also in saliva, the supragingival biofilm and advanced carious lesions $[34,35]$. In the oral biofilm in particular, the bacterial count of Lactobacillus species increases when the $\mathrm{pH}$ in the oral cavity decreases following carbohydrate metabolism [36].

Caries is characterized by demineralization of tooth enamel in the presence of high concentrations of organic acids that are produced by different species in the oral biofilm [37]. Commonly initial carious lesions develop under the influence of cariogenic bacteria, e.g. mutans streptococci and with the progression of a carious lesion the typical microflora shifts towards more lactobacilli and Bifidobacterium spp. as well as other acidogenic and aciduric species [38]. Indeed, various Lactobacillus species have been reported in carious lesions [35]. Lactobacillus fermentum has been found in childhood caries [39,40] and Lactobacillus casei/paracasei as well as Lactobacillus rhamnosus have been reported in root caries of elderly patients [41]. Molecular and culture analysis of advanced carious dentine revealed the presence of Lactobacillus gasseri, Lactobacillus johnsonii, Lactobacillus delbrueckii, Lactobacillus ultunensis, L. crispatus, L. fermentum, Lactobacillus salivarius, Lactobacillus casei/ rhamnosus and other related species $[4,42]$.

Despite the scientific consensus about the significance of the genus Lactobacillus for the human host, its species identification still poses several difficulties. Phenotypic detection criteria proved to be unreliable [43], e.g. in distinguishing between Lactobacillus acidophilus and closely related species $[9,10,44,45]$. It was not until molecular identification techniques had been applied that six distinct vaginal Lactobacillus species have been identified that had been classified as L. acidophilus on the basis of phenotypic characteristics before. Up to now, L. iners, as a very common vaginal species, was only described and classified in 1999 with 16S rRNA gene sequencing [14]. L. iners can indicate an abnormal flora or the tendency towards BV, e.g. during pregnancy. In general, clear species identification has only been possible using culture-independent methods not based on phenotypic criteria.

Precise species identification is indispensable in light of the findings that different Lactobacillus species can have very diverse effects on the host concerning pathogenesis of $\mathrm{BV}$ or triggering health-promoting mechanisms, e.g. stabilizing a healthy vaginal flora. Also tools for correct species identification are needed to further elucidate oral pathogenic processes, e.g. the development of advanced caries.

MALDI-TOF-MS analysis is a method which has been used for microbial species identification in routine laboratories for the last decade [46-50]. It holds the potential to provide a fast method for species identification of Lactobacillus isolates and would therefore facilitate beneficial monitoring of the vaginal flora. Yet the use of MALDI-TOF MS analysis has not been reported for the species identification of vaginal lactobacilli so far. $16 \mathrm{~S}$ rDNA-sequencing has been used for routine clinical diagnostics of bacterial species in infectious diseases as well $[9,20,51]$ and provides a highly discriminative comparison method.

To date there is no study comparing MALDI-TOF MS analysis and $16 \mathrm{~S}$ rDNA-sequencing for the identification of Lactobacillus species, isolated from clinical vaginal and oral samples. Therefore this study aimed to assess the capability of MALDI-TOF MS analysis for accurate species identification and the applicability of this method.

\section{Methods}

\section{Bacterial strains}

A total of 77 lactobacilli isolates gained from vaginal samples were used. The vaginal samples were obtained within a gynaecological examination with a speculum using sterile swabs (Sarstedt, Nümbrecht, Germany) that were placed in transport medium. The age range of the subjects was 20-69 (with only 1 subject beyond the reproductive age). All subjects gave their written informed consent to the study protocol. In addition, 21 lactobacilli isolates were collected from supragingival plaque samples and saliva samples. The oral samples were taken from unstimulated saliva and supragingival plaque of healthy male and female volunteers (aged 23-45) that had given their written informed consent to the study protocol. Prior to sampling, a detailed clinical oral examination was performed by a dentist, DMFT values were collected and saliva flow rates were measured at $1.2 \pm 0.2$. The 
preconditions for the volunteers were: Healthy dental status, i.e. low DMFT values (decayed, missing, filled teeth) of $0-5$, non-smokers, no severe systemic disease, no diseases of the salivary glands, no pregnancy or lactation, no use of antibiotics or local antimicrobial mouth rinses (e.g. Chlorhexidine) within the last month and no participation in another study. The supragingival plaque samples were treated for $30 \mathrm{~s}$ in an ultrasonic bath on ice, and dilution series of the samples were prepared as described by Al-Ahmad [52].

Additionally, 10 Lactobacillus reference strains from the German Collection of Microorganisms and Cell Cultures (DSMZ, Braunschweig, Germany) were tested.

All bacterial strains and isolates were cultivated on Columbia blood agar plates (CB; Oxoid, Wesel, Germany), Yeast-cysteine blood agar plates (HCB-agar; BectonDickinson, Nürnberg, Germany) and MRS agar plates (deMan Rogosa and Sharpe agar; Merck, Darmstadt, Germany). Cultivation was performed aerobically on CB and MRS agar for up to 7 days to capture slow-growing lactobacilli and anaerobically on HCB agar for 3 days (anaerobic chamber, GENbox BioMérieux, Nürtingen, Germany).

\section{MALDI-TOF sample preparation and MS analysis}

Without a detailed extraction step, bacteria from single colonies were used for MALDI-TOF analysis in a MALDI Biotyper Microflex LT (Bruker Daltonik, Bremen, Germany). For the acquisition of the mass spectra the authors followed the manufacturer's recommendations. The mass spectra were acquired within less than 5 minutes including the sample preparation. The BioTyper 3.0 software compared the obtained spectra with a reference database containing 3740 reference spectra (representing 319 genera and 1,946 species) and expressed the resulting similarity value as a log score. A score of $\geq 2.000$ indicated identification on the species level, a score of $\geq 1.700$ indicated identification on the genus level whereas any score under 1.700 meant no significant similarity of the obtained spectrum with any database entry. If the results were questionable, the procedure was repeated.

\section{DNA-extraction, genotypic analysis with 16S rDNA sequencing and phylogenetic analysis}

Material of pure cultures was used to extract total bacterial DNA. The colony was placed in $60 \mu \mathrm{l}$ PBS buffer and $240 \mu \mathrm{l}$ lysis buffer B (1\% Triton X-100 (Sigma-Aldrich, Taufkirchen, Germany), 0.5\% Tween 20 (Merck, Darmstadt, Germany), 10 mM TrisHCl (AppliChem, Darmstadt, Germany), 1 mM EDTA (SERVA, Heidelberg, Germany), in ultrapure water (Merck, Darmstadt, Germany), $\mathrm{pH}$ 8.0) were added. The solution was heated and incubated at $95{ }^{\circ} \mathrm{C}$ for $15 \mathrm{~min}$. Then the lysed cells were centrifuged at $14.000 \mathrm{rpm}$ for $5 \mathrm{~min}$ and the supernatant was transferred to a new $1.5 \mathrm{ml}$ vial and used for PCR.

A 1233 bp fragment of the $16 \mathrm{~S}$ rDNA was amplified using the following primers:

\section{TP16U1: AGAGTTTGATCMTGGCTCAG and RT16U6: ATTGTAGCACGTGTGTNGCCC [53].}

The reaction mixture of $50 \mu \mathrm{l}$ contained $25 \mu \mathrm{l}$ PCR-Mastermix (Qiagen, Hilden, Germany, including PCR Buffer, deoxyribonucleoside triphosphates and HotStar Taq DNA-Polymerase), $2.5 \mathrm{mM} \mathrm{MgCl}_{2}$ (Qiagen, Hilden, Germany) and 300nM of each primer. The PCR cycling conditions consisted of an initial denaturation step at $94^{\circ} \mathrm{C}$ for $2 \mathrm{~min}$, followed by 30 cycles with denaturation at $92^{\circ} \mathrm{C}$ for $1 \mathrm{~min}$, annealing at $55^{\circ} \mathrm{C}$ at $1 \mathrm{~min}$ and extension at $72^{\circ} \mathrm{C}$ for $1.5 \mathrm{~min}$, with a final extension step at $72^{\circ} \mathrm{C}$ for $5 \mathrm{~min}$. A no-template control and a positive control were included in each set of PCR reactions. Amplicons were analyzed by electrophoresis on a $1.5 \%$ agarose gel and positive reactions were used for sequencing.

The 16S rDNA-PCR products were purified before sequencing, using GFX PCR DNA and Gel Band Purification Kit (GE-Healthcare, Munich, Germany). Subsequently, Cycle Sequencing was performed on a 3130 Genetic Analyzer (Applied Biosystems, Life Technologies $\mathrm{GmbH}$, Darmstadt, Germany) applying the BigDye Terminator Cycle Sequencing Kit (Applied Biosystems, Darmstadt, Germany).

The sequence data obtained was visually proofread and edited and then compared to the public sequence databases, Genbank, EMBL and DDBJ using the BLAST program. The program was run through the server hosted by the National Center for Biotechnology Information NCBI (http://blast.ncbi.nlm.nih.gov/Blast.cgi) [54,55]. Sequences with $a \geq 98 \%$ match to a database sequence were considered to be of the same species as the one with the highest similarity and score bits. Additionally, all 16S rDNA sequences were compared with the database RIDOM (Ribosomal Differentiation of Microorganisms, Ridom $\mathrm{GmbH}$, Münster, Germany, accessed through the website www.ridomrdna.de).

The 16 s-rDNA sequences obtained were used for further comparative sequence analysis and phylogenetic analysis using the tools implemented in the software package ARB [56]. To implement the obtained sequences the reference dataset SSURef 111 SILVA NR 98040812 from the SILVA project [57] was used. Alignments were performed using the SINA Aligner plugin [58]. After manual correction of the alignment, a phylogenetic tree was constructed with the ARB Neighbor joining method applying the Jukes-Cantor correction and with the Maximum Parsimony and Maximum likelihood 
methods. For the Neighbor joining tree, bootstrapping was calculated based on 1000 replicates. Partial sequences were added without allowing changes of the tree topology by use of the ARB "parsimony interactive" method.

\section{Data deposition}

The partial $16 \mathrm{~S}$ rDNA sequences supporting the results were deposited in the NCBI Genbank database under the accession numbers KM250382-250423.

The data of the phylogenetic analysis are available from the Dryad Digital Repository: http://doi.org/10.5061/ dryad.78vs2.

\section{Results}

\section{Lactobacilli collection strains}

As a preliminary test of the applicability of MALDI TOF mass spectrometry for the identification of Lactobacillus species, a set of 10 reference strains were analyzed. The MALDI-TOF MS analysis gave correct results for all strains. The strains and results are listed in Table 1.

\section{Identification of vaginal and oral isolates with MALDI-TOF MS analysis and 16S rDNA analysis Vaginal isolates}

A total of 77 vaginal isolates were tested with MALDI-TOF MS analysis and genotypic analysis using 16S rDNA amplicon sequencing. The isolates belonged to 15 different lactobacilli species and both methods gave concordant results for 75 vaginal isolates, i.e. $97.4 \%$. The most abundant species were L. iners, L. crispatus, L. jensenii and L. gasseri, the results are summarized in Table 2 . The discordantly or insufficiently identified lactobacilli are listed in Table 3. In addition to 2 of the 5 discordantly identified samples, another 15 samples that gave concordant results with

Table 1 Reference strains tested with MALDI-TOF MS analysis

\begin{tabular}{|c|c|c|c|}
\hline Species & $\begin{array}{l}\text { Culture collection } \\
\text { number* }\end{array}$ & MALDI-TOF MS & $\begin{array}{l}\text { BioTyper log } \\
\text { score }\end{array}$ \\
\hline L. casei & DSM 20011 & L. casei** & 2.267 \\
\hline L. paracasei & DSM 20020 & L. paracasei & 2.080 \\
\hline L. fermentum & DSM 20052 & L. fermentum ${ }^{* *}$ & 1.974 \\
\hline L. brevis & DSM 20054 & L. brevis** & 2.258 \\
\hline L. delbrückii & DSM 20074 & L. delbrückii** & 2.097 \\
\hline L. plantarum & DSM 20174 & L. plantarum ${ }^{* *}$ & 2.303 \\
\hline L. acidophilus & DSM 20242 & L. acidophilus & 2.337 \\
\hline L. gasseri & DSM 20243 & L. gasseri** & 2.341 \\
\hline L. salivarius & DSM 20555 & L. salivarius ${ }^{* *}$ & 2.170 \\
\hline L. jensenii & DSM 20557 & L. jensenii ${ }^{* *}$ & 2.089 \\
\hline
\end{tabular}

*DSM: German collection of Microorganisms and Cell Cultures (DSMZ, Braunschweig, Germany).

** Type strains.
Table 2 Identification of lactobacilli isolates with MALDI-TOF MS and 16S rDNA-analysis: Vaginal isolates

\begin{tabular}{lccc}
\hline Species & 16S rDNA-analysis & MALDI-TOF-MS & $\begin{array}{l}\text { Concordance } \\
\text { [\%] }\end{array}$ \\
\hline L. iners & 18 & 18 & 100 \\
L. crispatus & 15 & 16 & 94 \\
L. jensenii & 14 & 15 & 93 \\
L. gasseri & 13 & 11 & 92 \\
L. fermentum & 4 & 4 & 100 \\
L. paracasei** & 3 & 3 & 100 \\
L. reuteri & 2 & 2 & 100 \\
L. delbrückii & 1 & 1 & 100 \\
L. mucosae & 1 & 1 & 100 \\
L. harbinensis & 1 & 1 & 100 \\
L. plantarum & 1 & 1 & 100 \\
L. rhamnosus & 1 & 1 & 100 \\
L. sakei & 1 & 1 & 100 \\
L. salivarius & 1 & 1 & 100 \\
L. vaginalis & 1 & 0 & - \\
Lactobacillus sp. & 0 & 1 & -
\end{tabular}

*Isolates identified as same species by both methods in relation to the total number of isolates of this species identified by $16 \mathrm{~S}$ rDNA sequencing.

** The $16 \mathrm{~S}$ rDNA-analysis result was $L$. paracasei/L. casei, both species were not distinguishable from each other.

both methods only yielded a MALDI-TOF BioTyper $\log$ score of 1.7-1.99, which indicates reliable identification on the genus level but only presumable identification on the species level. However, the resulting species were largely in agreement with the results of the sequencing. For three samples the differentiation between $L$. paracasei and $L$. casei by $16 \mathrm{~S}$ rDNA analysis was not possible due to the high sequence similarity of both species. Another two samples resulted in sequence data that could not be evaluated.

\section{Oral isolates}

A total of 21 oral isolates were tested with MALDI-TOF MS analysis and genotypic analysis using 16S rDNA amplicon sequencing. 19 isolates $(90.5 \%)$ yielded concordant results with both methods (Table 4). One of the two discordant samples was identified as $L$. fermentum with 16S rDNA analysis but as L. salivarius with MALDI-TOF MS analysis, the other one as Lactobacillus helveticus with 16S rDNA analysis and Lactobacillus ultunensis (BioTyper log score 1.71) with MALDI-TOF MS analysis (Table 3). Altogether three samples yielded BioTyper log scores between 1.85-1.97 which indicates reliable identification only on the genus level.

Overall, the oral isolates represented 11 different species with L. salivarius, L. gasseri, L. rhamnosus and L. paracasei identified in more than one sample. 
Table 3 Discordantly identified vaginal and oral isolates

\begin{tabular}{|c|c|c|c|c|}
\hline Sample Number & origin & 16S rDNA-analysis & MALDI-TOF-MS & BioTyper log score \\
\hline 12015 & vaginal & L. gasseri & L. crispatus & $1.891^{*}$ \\
\hline 13015 & vaginal & L. gasseri & L. jensenii & $1.944^{*}$ \\
\hline 12503 & vaginal & L. vaginalis & Lactobacillus sp. & 2.276 \\
\hline m113/6spbv1 & oral & L. fermentum & L. salivarius & 2.037 \\
\hline m114/10spv15 & oral & L. helveticus & L. ultunensis & $1.715^{*}$ \\
\hline
\end{tabular}

* $\log$ score $\leq 2.0$ (and $\geq 1.7$ ) indicates only reliable detection on the genus level, not the species level.

\section{Comparison of genotypic and MALDI-TOF analysis for vaginal and oral isolates}

Overall, nineteen different Lactobacillus species were identified, the most abundant species found in the vaginal samples were L. iners, L. crispatus, L. jensenii and $L$. gasseri, whereas oral samples (saliva and supragingival plaque) were dominated by L. salivarius, L. gasseri, $L$. rhamnosus and L. paracasei. Altogether 94 out of 98 lactobacilli isolates were analyzed accordantly with both methods, i.e. $96 \%$. The concordance of the methods with reference to the different Lactobacillus species and the origin of the isolates is shown in Tables 2 and 4. The detected lactobacilli belonged to six different phylogenetic groups [59], namely the Lactobacillus acidophilus group, Lactobacillus reuteri group, Lactobacillus buchneri group, Lactobacillus plantarum group and Lactobacillus sakei/ casei group. The phylogenetic analysis of $16 \mathrm{~S}$ rDNA sequences of selected oral and vaginal isolates performed with the ARB software is shown in Figure 1. The phylogenetic analysis showed that oral isolates cluster together and most of them are very close to the vaginal

Table 4 Identification of lactobacilli isolates with MALDI-TOF MS and 16S rDNA-analysis: Oral isolates

\begin{tabular}{lccc}
\hline Species & 16S rDNA-analysis & MALDI-TOF-MS & $\begin{array}{c}\text { Concordance } \\
{[\%]^{*}}\end{array}$ \\
\hline L. salivarius & 3 & 4 & 75 \\
L. gasseri & 3 & 3 & 100 \\
L. rhamnosus & 3 & 3 & 100 \\
L. paracasei** & 3 & 3 & 100 \\
L. fermentum & 2 & 1 & 50 \\
L. oris & 2 & 2 & 100 \\
L. crispatus & 2 & 2 & 100 \\
L. kalixensis & 1 & 1 & 100 \\
L. reuteri & 1 & 1 & 100 \\
L. helveticus & 1 & 0 & - \\
L. ultunensis & 0 & 1 & -
\end{tabular}

* Isolates identified as same species by both methods in relation to the total number of isolates of this species identified by $16 \mathrm{~S}$ rDNA sequencing.

**The $16 \mathrm{~S}$ rDNA-analysis result was $L$. paracasei/L. casei, both species were not distinguishable from each other.

***The BioTyper log score was 1.71, reliable identification on genus level, but not on species level, repetition of the samples resulted in L. acidophilus with a $\log$ score of 1.87 . isolates. One oral isolate that was identified as L. helveticus with 16S rDNA analysis was identified as L. ultunensis / L. acidophilus by MALDI TOF MS analysis. In the phylogenetic analysis this sequence clustered with the $L$. helveticus sequences in agreement with the $16 \mathrm{~S}$-sequencing result. Analyzed sequences were deposited in the GenBank database.

\section{Discussion}

In this study MALDI-TOF MS analysis was compared with $16 \mathrm{~S}$ rDNA sequencing to identify 98 lactobacilli isolates from vaginal and oral samples on the species level with the aim of evaluating the potential of mass spectra analysis as a specific and fast detection method. The two methods yielded $96 \%$ concordant results. Combining isolates from different clinically relevant sources further affirms the validity of the method. Other studies report similar percentage concordance comparing mass spectrometry with different genotypic methods, e.g. Callaway et al. 93\% [35] and Angelakis et al. 92\% [60], or somewhat lower values, e.g. Duskova et al. 74\% [43] and Dec et al. 88.5\% [61].

The MALDI-TOF MS analysis demonstrated accurate species-level identification for those Lactobacillus species that are of importance for vaginal health as well as those that can indicate the beginning stages of BV. Equally, species associated with progressive caries were identified correctly. Even though MALDI-TOF MS analysis has been used to analyze bacterial species from clinical specimens for nearly two decades $[46,62,63]$ it has not been thoroughly evaluated for vaginal lactobacilli. To date the method has been applied to identify lactobacilli originating from food to the species level $[43,60,61,64]$ and recently attempts have been made to even differentiate subspecies of Lactobacillus brevis that are known to cause beer spoilage [65]. Furthermore, Callaway [35] has reported successful species identification of lactobacilli from carious dentine.

$16 \mathrm{~S}$ rDNA sequencing, which was applied as the comparison method, is regarded as an established method for exact species identification and is also applied for clinical diagnostic purposes [66]. Given the known difficulties of exact species identification of lactobacilli by means of phenotypic methods $[14,43,67]$, studies on the taxonomy 


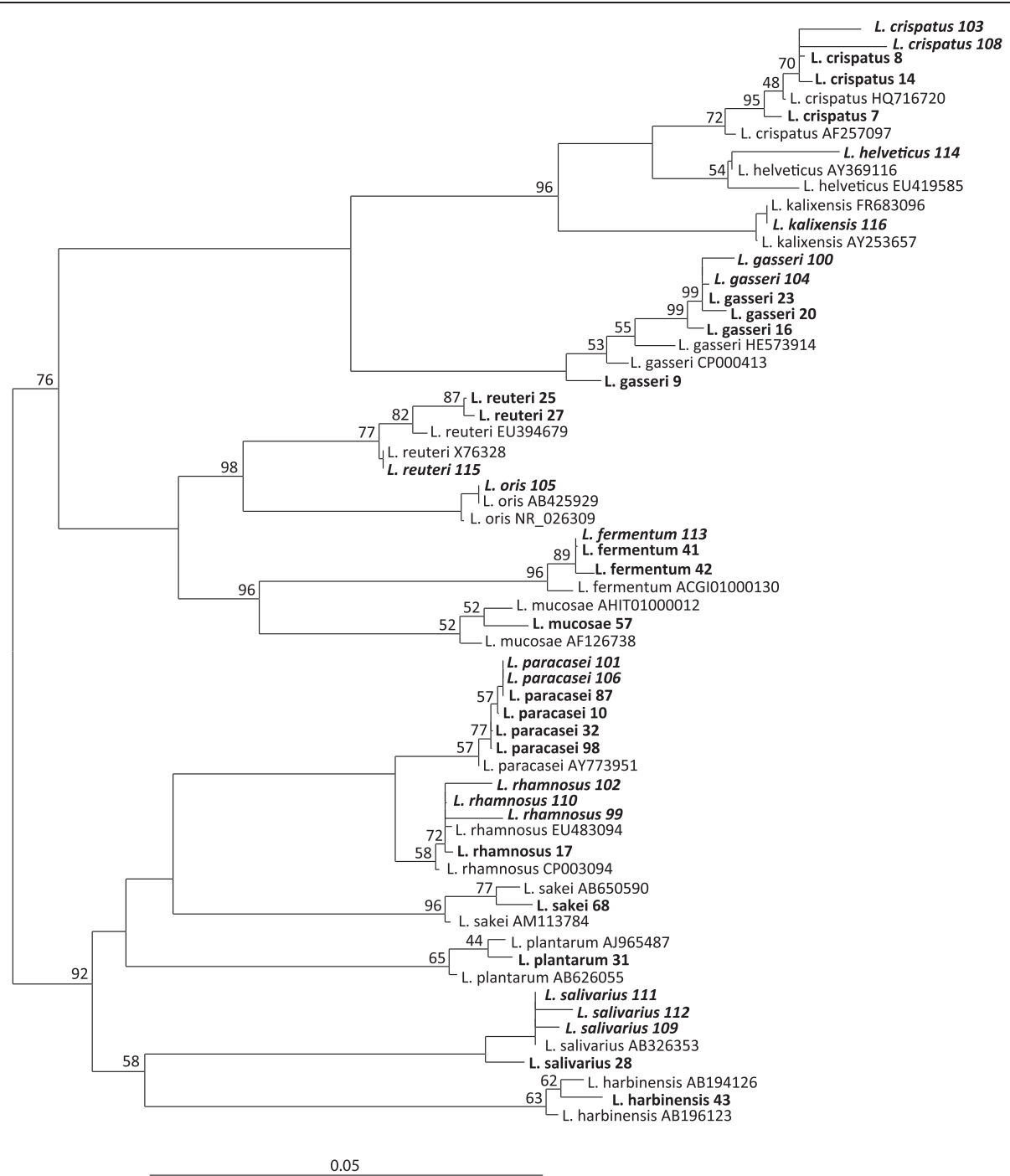

Figure 1 Phylogenetic tree based on the analysis of $16 \mathrm{~S}$ rRNA gene sequences derived from oral and vaginal isolates. $16 \mathrm{~S}$ rDNA sequences were aligned using the SINA plugin (ARB software package) and distances were calculated using the neighbor-joining method with Jukes-Cantor correction. Reference sequences with accession numbers are in regular type, sample sequences of vaginal isolates in bold type and of oral isolates in bold/italics type. Sample strains are named according to their identification by $16 \mathrm{~S}$ rDNA sequencing results. Bootstrap values (from 1000 replicates) greater than 40\% are shown at the branch points. The bar indicates 5\% sequence divergence.

of lactobacilli or on Lactobacillus species identification have been performed using molecular methods based on 16S sequence differences, such as Amplified Ribosomal DNA Restriction Analysis (ARDRA) [67-69], Ribotyping [70,71], 16S rDNA sequencing and others $[3,9,18,72]$. Identifying lactobacilli employing $16 \mathrm{~S}$ rDNA sequencing has shown to be a highly discriminative method and has revealed new species, e.g. L. iners [73]. Nevertheless, it is not an infallible method and it has failed to differentiate between $L$. case $i$ and $L$. paracase $i$ in our study, due to the substantial similarities of their $16 \mathrm{~S}$ rDNA genes. In some cases, Lactobacillus species can only be correctly identified combining several genotypic methods [61].
Overall, our study demonstrated a high discriminatory power of the mass spectra analysis for the identification of Lactobacillus species that are relevant indicators for vaginal health, e.g. $L$. crispatus, $L$. gasseri and $L$. jensenii as well as $L$. iners, a species that is frequently detected in vaginal flora exhibiting a tendency towards BV $[1,22]$. Before the application of molecular techniques L. crispatus, $L$. jensenii and L. gasseri, all belonging to the L. acidophilusgroup had not been differentiated as distinct species in the past [9]. In our study, all these species were correctly identified by MALDI-TOF MS analysis. Mass spectra analysis proved equally suitable for the detection of Lactobacillus species associated with different forms 
of caries. The species found most frequently in saliva and supragingival plaque samples in this study, namely $L$. salivarius, L. gasseri, L. rhamnosus and L. paracasei concur with those species found in carious lesions [35,72].

Apart from the high accuracy of the method, the rapidity of the analysis is particularly advantageous for the application in clinical laboratories. After obtaining the bacterial isolates the acquisition of the mass spectra, including the sample preparation, takes less than five minutes compared to several hours or even several days by molecular or phenotypic analysis depending on the respective method. Thus the biochemical identification of lactobacilli with the commercial API 50 CHL system (Biomérieux, Marcy l' Etoile, France) includes fifty reactions and takes an additional $48 \mathrm{~h}$ of incubation time after obtaining pure cultures.

Most of the differing results with the two compared methods can be explained: Two samples, identified as L. gasseri with $16 \mathrm{~S}$ rDNA analysis were identified as L. crispatus and L. jensenii with MALDI-TOF analysis respectively. For both samples, the BioTyper log score was below 2.0, indicating uncertain species identification. Also, L. gasseri, L. jensenii and L. crispatus are all phylogenetically closely related species. The same applies to one oral sample that was identified as $L$. helveticus genotypically and as L. ultunensis / L. acidophilus by mass spectrometry. Both species are closely related and again the BioTyper log score was below 2.0. The phylogenetic analysis affirms the sequence similarity with $L$. helveticus.

One of the main weaknesses of the mass spectrometry method revealed by our experiments was the frequent occurrence of MALDI-BioTyper log scores between 1.68 and 1.99. Values between 1.7 and 2.0 indicate correct genus identification but only presumptive species identification. Yet from 19 samples showing log scores $<2.0$, the vast majority of the presumed species by the BioTyper software (16 samples) agreed with the results achieved by $16 \mathrm{~S}$ rDNA sequencing, suggesting that despite the low log score the MALDI-TOF MS identification on the species level was correct. This outcome of the mass spectrometry might be improved by extending the BioTyper reference database, as Duskova [43] has stated before. Evidently, the MALDI BioTyper database and corresponding databases of other MALDI-TOF devices will be continuously expanded with the increase of its applications.

\section{Conclusions}

Apart from the above mentioned minor drawback, our study demonstrates the excellent applicability of mass spectra analysis to identify lactobacilli to the species level. The MALDI-TOF MS analysis, e.g. using the commercially available MALDI BioTyper system, provides a fast as well as cost-effective method, which proves to be especially relevant considering the labor intensity of genotypic and phenotypic methods characteristic for Lactobacillus species identification. Accurate species identification for clinically relevant species in relation to vaginal health, BV and caries can be achieved in a short time once isolates are obtained. Since it has been shown in recent years that different Lactobacillus species have different protective functions for the vaginal flora, specific methods for species-level identification are necessary and profitable for monitoring vaginal health during pregnancy and other stages. MALDI-TOF MS analysis can also facilitate analysis of lactobacilli involved in pathological processes, e. g. progressive caries. Beyond that scope, mass spectra analysis can also help to analyze Lactobacillus strains that hold the potential for probiotic application.

\section{Consent}

This study was approved by the Ethics Committee of the Albert Ludwigs-University Freiburg (42/08).

\section{Competing interests}

The authors declare that they have no competing interests.

\section{Authors' contributions}

ACA analyzed and interpreted the data, performed the phylogenetic analysis and drafted the manuscript. SM carried out the molecular methods and the MALDI-TOF MS experiments and analyzed the data. SC participated in the design of the study, the acquisition of the samples and the drafting of the manuscript. CA participated in the design of the study. $\mathrm{KL}$ and $\mathrm{HE}$ revised the manuscript critically for important intellectual content. AA participated in the design of the study and revised the manuscript critically for important intellectual content. All authors read and approved the final manuscript.

\section{Authors' information}

Andreas Clad posthumous.

\section{Acknowledgements}

The authors would like to thank Heike Wölk and Annette Wittmer for their excellent technical support as well as Dr. Michael Schmid for helpful, informal ARB support.

\section{Author details}

${ }^{1}$ Department of Operative Dentistry and Periodontology, Albert-Ludwigs-University, Hugstetter Strasse 55, Freiburg, Germany. ${ }^{2}$ Department of Hygiene and Microbiology, Albert-Ludwigs-University, Hermann-Herder-Str. 11, Freiburg, Germany. ${ }^{3}$ Department for Gynaecology, Medical Center, Albert-Ludwigs-University, Hugstetter Strasse 55, Freiburg, Germany.

Received: 23 July 2014 Accepted: 25 November 2014 Published online: 14 December 2014

\section{References}

1. Balashov SV, Mordechai E, Adelson ME, Sobel JD, Gygax SE: Multiplex quantitative polymerase chain reaction assay for the identification and quantitation of major vaginal lactobacilli. Diagn Microbiol Infect Dis 2014, 78:321-327.

2. Tannock GW: A special fondness for lactobacilli. Appl Environ Microbiol 2004, 70:3189-3194.

3. Pavlova SI, Kilic AO, Kilic SS, So JS, Nader-Macias ME, Simoes JA, Tao L: Genetic diversity of vaginal lactobacilli from women in different countries based on 16S rRNA gene sequences. J Appl Microbiol 2002, 92:451-459. 
4. Munson MA, Banerjee A, Watson TF, Wade WG: Molecular analysis of the microflora associated with dental caries. J Clin Microbiol 2004, 42:3023-3029.

5. O'Callaghan J, O'Toole PW: Lactobacillus: host-microbe relationships. Curr Top Microbiol Immunol 2013, 358:119-154.

6. Redondo-Lopez V, Cook RL, Sobel JD: Emerging role of lactobacilli in the control and maintenance of the vaginal bacterial microflora. Rev Infect Dis 1990, 12:856-872.

7. Reid G, McGroarty JA, Tomeczek L, Bruce AW: Identification and plasmid profiles of Lactobacillus species from the vagina of 100 healthy women. FEMS Immunol Med Microbiol 1996, 15:23-26.

8. Antonio MA, Rabe LK, Hillier SL: Colonization of the rectum by Lactobacillus species and decreased risk of bacterial vaginosis. J Infect Dis 2005, 192:394-398.

9. Vasquez A, Jakobsson T, Ahrne S, Forsum U, Molin G: Vaginal lactobacillus flora of healthy Swedish women. J Clin Microbiol 2002, 40:2746-2749.

10. Zhou X, Bent SJ, Schneider MG, Davis CC, Islam MR, Forney L: Characterization of vaginal microbial communities in adult healthy women using cultivation-independent methods. Microbiology 2004, 150:2565-2573.

11. Zhou X, Brown CJ, Abdo Z, Davis CC, Hansmann MA, Joyce P, Foster JA, Forney $L$ : Differences in the composition of vaginal microbial communities found in healthy Caucasian and black women. Isme j 2007, 1:121-133.

12. Linhares IM, Giraldo PC, Baracat EC: [New findings about vaginal bacterial flora]. Rev Assoc Med Bras 2010, 56:370-374

13. Ma B, Forney $L$, Ravel J: Vaginal microbiome: rethinking health and disease. Annu Rev Microbiol 2012, 66:371-389.

14. Lamont RF, Sobel JD, Akins RA, Hassan SS, Chaiworapongsa T, Kusanovic JP, Romero R: The vaginal microbiome: new information about genital tract flora using molecular based techniques. Bjog 2011, 118:533-549.

15. Ravel J, Gajer P, Abdo Z, Schneider GM, Koenig SS, McCulle SL, Karlebach S, Gorle R, Russell J, Tacket CO, Brotman RM, Davis CC, Ault K, Peralta L, Forney LJ: Vaginal microbiome of reproductive-age women. Proc Natl Acad Sci U S A 2011, 108(Suppl 1):4680-4687.

16. Yoshimura K, Morotomi N, Fukuda K, Nakano M, Kashimura M, Hachisuga T, Taniguchi $\mathrm{H}$ : Intravaginal microbial flora by the $16 \mathrm{~S}$ rRNA gene sequencing. Am J Obstet Gynecol 2011, 205:235.e231-239.

17. Nardis C, Mosca L, Mastromarino P: Vaginal microbiota and viral sexually transmitted diseases. Ann lg 2013, 25:443-456.

18. Pendharkar S, Magopane T, Larsson PG, de Bruyn G, Gray GE, Hammarstrom $\mathrm{L}$, Marcotte $\mathrm{H}$ : Identification and characterisation of vaginal lactobacilli from South African women. BMC Infect Dis 2013, 13:43.

19. Boris $S$, Barbes $C$ : Role played by lactobacilli in controlling the population of vaginal pathogens. Microbes Infect 2000, 2:543-546.

20. Fredricks DN, Fiedler TL, Marrazzo JM: Molecular identification of bacteria associated with bacterial vaginosis. N Engl J Med 2005, 353:1899-1911.

21. Donders GG: Definition and classification of abnormal vaginal flora. Best Pract Res Clin Obstet Gynaecol 2007, 21:355-373.

22. Verstraelen $H$, Verhelst R, Claeys $G$, De Backer $E$, Temmerman M, Vaneechoutte M: Longitudinal analysis of the vaginal microflora in pregnancy suggests that L. crispatus promotes the stability of the normal vaginal microflora and that L. gasseri and/or L. iners are more conducive to the occurrence of abnormal vaginal microflora. BMC Microbiol 2009, 9:116.

23. Allsworth JE, Peipert JF: Prevalence of bacterial vaginosis: 2001-2004 National Health and Nutrition Examination Survey data. Obstet Gynecol 2007, 109:114-120

24. Peipert JF, Montagno AB, Cooper AS, Sung CJ: Bacterial vaginosis as a risk factor for upper genital tract infection. Am J Obstet Gynecol 1997, 177:1184-1187.

25. Martin HL, Richardson BA, Nyange PM, Lavreys L, Hillier SL, Chohan B, Mandaliya K, Ndinya-Achola JO, Bwayo J, Kreiss J: Vaginal lactobacilli, microbial flora, and risk of human immunodeficiency virus type 1 and sexually transmitted disease acquisition. J Infect Dis 1999, 180:1863-1868.

26. Pybus $V$, Onderdonk AB: Microbial interactions in the vaginal ecosystem, with emphasis on the pathogenesis of bacterial vaginosis. Microbes Infect 1999, 1:285-292

27. Harmanli $\mathrm{OH}$, Cheng GY, Nyirjesy P, Chatwani A, Gaughan JP: Urinary tract infections in women with bacterial vaginosis. Obstet Gynecol 2000 95:710-712.
28. Wang Y, Chen C, Ai L, Zhou F, Zhou Z, Wang L, Zhang H, Chen W, Guo B: Complete genome sequence of the probiotic Lactobacillus plantarum ST-III. J Bacterio/ 2011, 193:313-314.

29. Reid G: Probiotic and prebiotic applications for vaginal health. J AOAC Int 2012, 95:31-34.

30. Marelli G, Papaleo E, Ferrari A: Lactobacilli for prevention of urogenital infections: a review. Eur Rev Med Pharmacol Sci 2004, 8:87-95.

31. Stapleton AE, Au-Yeung M, Hooton TM, Fredricks DN, Roberts PL, Czaja CA Yarova-Yarovaya Y, Fiedler T, Cox M, Stamm WE: Randomized, placebo-controlled phase 2 trial of a Lactobacillus crispatus probiotic given intravaginally for prevention of recurrent urinary tract infection. Clin Infect Dis 2011, 52:1212-1217.

32. Mastromarino $P$, Vitali $B$, Mosca L: Bacterial vaginosis: a review on clinical trials with probiotics. New Microbiol 2013, 36:229-238.

33. Verstraelen $H$, Swidsinski A: The biofilm in bacterial vaginosis: implications for epidemiology, diagnosis and treatment. Curr Opin Infect Dis 2013, 26:86-89.

34. de Soet JJ, de Graaff J: Microbiology of carious lesions. Dent Update 1998, 25:319-324.

35. Callaway A, Kostrzewa M, Willershausen B, Schmidt F, Thiede B, Kupper H, Kneist S: Identification of Lactobacilli from deep carious lesions by means of species-specific PCR and MALDI-TOF mass spectrometry. Clin Lab 2013, 59:1373-1379.

36. Marsh PD, Bradshaw DJ: Physiological approaches to the control of oral biofilms. Adv Dent Res 1997, 11:176-185.

37. van Houte J: Role of micro-organisms in caries etiology. J Dent Res 1994, 73:672-681

38. Takahashi N, Nyvad B: The role of bacteria in the caries process: ecological perspectives. J Dent Res 2011, 90:294-303.

39. Becker MR, Paster BJ, Leys EJ, Moeschberger ML, Kenyon SG, Galvin JL, Boches SK, Dewhirst FE, Griffen AL: Molecular analysis of bacterial species associated with childhood caries. J Clin Microbiol 2002, 40:1001-1009.

40. Tanner AC, Mathney JM, Kent RL, Chalmers NI, Hughes CV, Loo CY, Pradhan $\mathrm{N}$, Kanasi E, Hwang J, Dahlan MA, Papadopolou E, Dewhirst FE: Cultivable anaerobic microbiota of severe early childhood caries. J Clin Microbiol 2011, 49:1464-1474.

41. Preza D, Olsen I, Willumsen T, Boches SK, Cotton SL, Grinde B, Paster BJ: Microarray analysis of the microflora of root caries in elderly. Eur J Clin Microbiol Infect Dis 2009, 28:509-517.

42. Chhour KL, Nadkarni MA, Byun R, Martin FE, Jacques NA, Hunter N: Molecular analysis of microbial diversity in advanced caries. J Clin Microbiol 2005, 43:843-849.

43. Duskova M, Sedo O, Ksicova K, Zdrahal Z, Karpiskova R: Identification of lactobacilli isolated from food by genotypic methods and MALDI-TOF MS. Int J Food Microbiol 2012, 159:107-114.

44. Klein G, Pack A, Bonaparte C, Reuter G: Taxonomy and physiology of probiotic lactic acid bacteria. Int J Food Microbiol 1998, 41:103-125.

45. Antonio MA, Hawes SE, Hillier SL: The identification of vaginal Lactobacillus species and the demographic and microbiologic characteristics of women colonized by these species. J Infect Dis 1999, 180:1950-1956.

46. Eigner U, Holfelder M, Oberdorfer K, Betz-Wild U, Bertsch D, Fahr AM: Performance of a matrix-assisted laser desorption ionization-time-of-flight mass spectrometry system for the identification of bacterial isolates in the clinical routine laboratory. Clin Lab 2009, 55:289-296.

47. Marklein G, Josten M, Klanke U, Muller E, Horre R, Maier T, Wenzel T, Kostrzewa M, Bierbaum G, Hoerauf A, Sahl HG: Matrix-assisted laser desorption ionization-time of flight mass spectrometry for fast and reliable identification of clinical yeast isolates. J Clin Microbiol 2009, 47:2912-2917.

48. Seng P, Drancourt M, Gouriet F, La Scola B, Fournier PE, Rolain JM, Raoult D: Ongoing revolution in bacteriology: routine identification of bacteria by matrix-assisted laser desorption ionization time-of-flight mass spectrometry. Clin Infect Dis 2009, 49:543-551

49. Christner M, Rohde H, Wolters M, Sobottka I, Wegscheider K, Aepfelbacher M: Rapid identification of bacteria from positive blood culture bottles by use of matrix-assisted laser desorption-ionization time of flight mass spectrometry fingerprinting. J Clin Microbio/ 2010, 48:1584-1591.

50. van Veen SQ, Claas EC, Kuijper EJ: High-throughput identification of bacteria and yeast by matrix-assisted laser desorption ionization-time of flight mass spectrometry in conventional medical microbiology laboratories. J Clin Microbiol 2010, 48:900-907. 
51. Harris KA, Hartley JC: Development of broad-range $16 \mathrm{~S}$ rDNA PCR for use in the routine diagnostic clinical microbiology service. J Med Microbiol 2003, 52:685-691.

52. Al-Ahmad A, Maier J, Follo M, Spitzmuller B, Wittmer A, Hellwig E, Hubner J, Jonas D: Food-borne enterococci integrate into oral biofilm: an in vivo study. J Endod 2010, 36:1812-1819.

53. Schuppler M, Mertens F, Schon G, Gobel UB: Molecular characterization of nocardioform actinomycetes in activated sludge by $16 \mathrm{~S}$ rRNA analysis. Microbiology 1995, 141(Pt 2):513-521.

54. Altschul SF, Gish W, Miller W, Myers EW, Lipman DJ: Basic local alignment search tool. J Mol Biol 1990, 215:403-410.

55. Altschul SF, Madden TL, Schaffer AA, Zhang J, Zhang Z, Miller W, Lipman DJ: Gapped BLAST and PSI-BLAST: a new generation of protein database search programs. Nucleic Acids Res 1997, 25:3389-3402.

56. Ludwig W, Strunk O, Westram R, Richter L, Meier H, Yadhukumar, Buchner A Lai T, Steppi S, Jobb G, Förster W, Brettske I, Gerber S, Ginhart AW, Gross O, Grumann S, Hermann S, Jost R, König A, Liss T, Lüssmann R, May M, Nonhoff B, Reichel B, Strehlow R, Stamatakis A, Stuckmann N, Vilbig A, Lenke M, Ludwig T, et al: ARB: a software environment for sequence data. Nucleic Acids Res 2004, 32:1363-1371.

57. Quast C, Pruesse E, Yilmaz P, Gerken J, Schweer T, Yarza P, Peplies J, Glockner FO: The SILVA ribosomal RNA gene database project: improved data processing and web-based tools. Nucleic Acids Res 2013, 41:D590-596.

58. Pruesse E, Peplies J, Glockner FO: SINA: accurate high-throughput multiple sequence alignment of ribosomal RNA genes. Bioinformatics 2012, 28:1823-1829.

59. Canchaya C, Claesson MJ, Fitzgerald GF, van Sinderen D, OToole PW: Diversity of the genus Lactobacillus revealed by comparative genomics of five species. Microbiology 2006, 152:3185-3196.

60. Angelakis E, Million M, Henry M, Raoult D: Rapid and accurate bacterial identification in probiotics and yoghurts by MALDI-TOF mass spectrometry. J Food Sci 2011, 76:M568-572.

61. Dec M, Urban-Chmiel R, Gnat S, Puchalski A, Wernicki A: Identification of Lactobacillus strains of goose origin using MALDI-TOF mass spectrometry and 16S-23S rDNA intergenic spacer PCR analysis. Res Microbiol 2014, 165:190-201.

62. Mazzeo MF, Sorrentino A, Gaita M, Cacace G, Di Stasio M, Facchiano A Comi G, Malorni A, Siciliano RA: Matrix-assisted laser desorption ionization-time of flight mass spectrometry for the discrimination of food-borne microorganisms. Appl Environ Microbiol 2006, 72:1180-1189.

63. Claydon MA, Davey SN, Edwards-Jones V, Gordon DB: The rapid identification of intact microorganisms using mass spectrometry. Nat Biotechnol 1996, 14:1584-1586.

64. Doan NT, Van Hoorde K, Cnockaert M, De Brandt E, Aerts M, Le Thanh B, Vandamme P: Validation of MALDI-TOF MS for rapid classification and identification of lactic acid bacteria, with a focus on isolates from traditional fermented foods in Northern Vietnam. Lett Appl Microbiol 2012, 55:265-273.

65. Kern CC, Vogel RF, Behr J: Differentiation of lactobacillus brevis strains using matrix-assisted-laser-desorption-ionization-time-of-flight mass spectrometry with respect to their beer spoilage potential. Food Microbiol 2014, 40:18-24.

66. Patel JB: $16 \mathrm{~S}$ rRNA gene sequencing for bacterial pathogen identification in the clinical laboratory. Mol Diagn 2001, 6:313-321.

67. Dimitonova SP, Bakalov BV, Aleksandrova-Georgieva RN, Danova ST: Phenotypic and molecular identification of lactobacilli isolated from vaginal secretions. J Microbiol Immunol Infect 2008, 41:469-477.

68. Pal K, Szen O, Kiss A, Naar Z: Comparison and evaluation of molecular methods used for identification and discrimination of lactic acid bacteria. J Sci Food Agric 2012, 92:1931-1936.

69. Moreira JL, Mota RM, Horta MF, Teixeira SM, Neumann E, Nicoli JR, Nunes AC: Identification to the species level of Lactobacillus isolated in probiotic prospecting studies of human, animal or food origin by 16S-23S rRNA restriction profiling. BMC Microbiol 2005, 5:15.

70. Zhong W, Millsap K, Bialkowska-Hobrzanska H, Reid G: Differentiation of lactobacillus species by molecular typing. Appl Environ Microbiol 1998, 64:2418-2423.

71. Giraffa G, Gatti M, Rossetti L, Senini L, Neviani E: Molecular diversity within Lactobacillus helveticus as revealed by genotypic characterization. Appl Environ Microbiol 2000, 66:1259-1265.
72. Yang R, Argimon S, Li Y, Gu H, Zhou X, Caufield PW: Determining the genetic diversity of lactobacilli from the oral cavity. J Microbiol Methods 2010, 82:163-169.

73. Falsen E, Pascual C, Sjoden B, Ohlen M, Collins MD: Phenotypic and phylogenetic characterization of a novel Lactobacillus species from human sources: description of Lactobacillus iners sp. nov. Int J Syst Bacteriol 1999, 49 Pt 1:217-221.

doi:10.1186/s12866-014-0312-5

Cite this article as: Anderson et al:: Rapid species-level identification of vaginal and oral lactobacilli using MALDI-TOF MS analysis and 16S rDNA sequencing. BMC Microbiology 2014 14:312.

\section{Submit your next manuscript to BioMed Central and take full advantage of:}

- Convenient online submission

- Thorough peer review

- No space constraints or color figure charges

- Immediate publication on acceptance

- Inclusion in PubMed, CAS, Scopus and Google Scholar

- Research which is freely available for redistribution

Submit your manuscript at www.biomedcentral.com/submit
C) Biomed Central 